\title{
Study on the Methods to Cultivate Students' Positive Mental Quality from the Perspective of Positive Psychology
}

\author{
Yi Zhang ${ }^{1}$ \\ ${ }^{1}$ Shaanxi University of Science \& Technology, Xi'an, Shaanxi, 710021 \\ 346591653@163.com
}

KEYWORDS: Positive Psychology Perspective; Positive Psychological Quality; Training Ways

\begin{abstract}
Positive psychology is a new subject that studies traditional psychology from a positive point of view. It mainly studies positive psychological quality of human, focusing on health and happiness and harmonious development of mankind. Students are at an important stage in the transition to adulthood, whether they have a good psychological quality, have a positive attitude to their future life has far-reaching significance. This article is from the perspective of positive psychology, to discuss ways to effectively improve the psychological quality of students actively.
\end{abstract}

\section{Introduction}

Positive psychological quality has become one of the important quality of students, it can effectively improve the psychological quality of college students maintain a positive, optimistic, confident of a good attitude in learning and life. However, due to the imperfections of family education and school education, college students are still causing some bad psychological quality, such as cognitive bias, prone to negative emotions, willpower, there is not enough motivation, the overall capacity of not high quality. These adverse psychologies affect quality mental health of college students, college students make in the future is difficult to face fierce social competition.

\section{The Concept of Positive Psychology}

Positive Mental quality is first proposed by Hillson and Made in 1999, and later seligman define positive psychological quality in "positive Introduction to Psychology" in his book: mental quality is a subjective sense of happiness, optimism, happiness and self-determination, and the like, and the virtues mental strength is a positive quality of the core, with a similar buffer role is to help people overcome mental illness powerful weapon. But after Christopher Peterson and George Vaillant quality through the study of positive psychology has established the following seven criteria: (1) positive mental quality is a trait with general stability across scenarios and over time; (2) positive psychological qualities can help yourself or others happier life; (3) positive mental receive moral quality assessment, rather than results; (4) when a person exhibits positive psychological quality, can inspire people around also revealed this trait; (5) positive psychological quality is supported by the power of civilization; (6) each person has a positive psychological quality has a recognized role model; (7) positive mental quality cannot be decomposed into other benefits.

Positive Mental quality is a person in the family education, school education and social environment gradually, with good stability. Positive psychological qualities inherent potential to inspire people, to help people with a positive attitude towards life, work difficulties and gradually 
achieves self-improvement. Positive psychology is the psychological quality of the body's internal characteristics, has the potential to be manifested in order to external stimuli, and therefore need people conscious self -discovery and culture.

\section{The Importance of Cultivating Positive Psychology}

In modern society, health is not only refers to physical health, and mental health. The two are complementary, interdependent relationship. Mental health help keep the body healthy, healthy body is also conducive to the promotion of mental health. Pay attention to cultivate a positive mental quality of college students, it remains an important measure to achieve healthy development.

When more positive psychological quality of college students, it will be less negative psychological quality, help students maintain a positive and optimistic mood. Modern psychological research shows that psychological quality positive psychological quality of the generated effectively adjust the body's nervous system, endocrine system, so that it is in good condition inside, and mental illness have good regulation and buffer.

It can play a positive psychological quality of college students to enhance the therapeutic effects of the disease. When college students due to illness treatment, quality can play a positive psychological "placebo" effect, even college students actually did not accept for the relief of pain medication, the situation will improve or alleviate the symptoms appear. Positive Mental quality so that students always an optimistic attitude to face the disease and maintain their emotional pleasure, which is very conducive to disease recovery.

Positive psychology that not all individuals have the potential ability through conscious mining and develop these capabilities, you can let people in appropriate conditions to maximize the full potential. Positive Psychology also found that positive psychological quality to make it easier for people to be successful. Mainly because people always have a positive quality of life, excited about the future, they are more willing to pursue success; people have a positive psychological qualities are more likely to come out negative emotions, timely adjust their positive face of difficulties, so it is easier to solve problems; people have a positive psychological quality is more creative and easier to stimulate their potential. And college students are in the best moment of the life state, and develop a positive mental quality can effectively enhance their level of knowledge, learning ability, creative ability, to make their own emotional and always maintain a good mental state, to help themselves to better complete the self-improvement and self-growth.

The so-called happiness, is that people under the internalized social standards of their quality of life, integrity, positive assessment of people satisfaction and comprehensive evaluation of all aspects of life, and the resulting positive emotion, dominant mental state. Positive psychological quality of college students depends on good family education, school education and social environment, so that students in difficulty or suffering against time to get spiritual and material help to increase the well-being of college students and the ability to face difficulties. University life or future social life, have positive psychological quality of college students easier access to other people's approval, establish good relationships with positive psychological quality of college students not only willing to take to help others, others will get help, so Students make sufficient sense of belonging and self-identity, effectively increasing the well-being of college students.

\section{The Methods to Effectively Cultivate Positive Psychology}

Positive cognitive ability to benefit students develop a positive and optimistic attitude, which guide students to develop a positive mental qualities. As a basis for cognitive ability, college 
students are objective and comprehensive knowledge themselves, to a correct understanding of other people and things. During positive self-awareness, community college students make a rational comparison. In society (university) interaction with others through their own evaluation or analysis of the behavior of some of the specific things, that can help correct and objective understanding of their own. In their reasonable comparison with others, we discover the advantages of others, their own lack of learning to learn from others, thus gradually improve themselves. In the process of self-awareness, the students should learn to accept yourself, accept their own shortcomings and correct view of their advantages. Only fully accept themselves, to accept others, in order to cultivate a certain inclusive, so that their better growth. In the university, teachers organize students to "my favorite people" discussions allow students to talk about their favorite person is like, have a kind of character and quality, help students develop slowly discover the advantages of the habit of thinking of others . In society, students can ask someone, "What is your view?" Or "Do you think it should be how to solve" such statements to know one thing different views, those views through understanding and analysis, people will gradually understand the advantages and benefits and to learn.

Frustration with the right objective cognitive self-awareness is equally important. Student experiences the simple life, not enough social experience, in the face of setbacks do not have sufficient capacity to respond and cognitive abilities. When they own setbacks, students should realize that setbacks and failures are common. When they can calm the face of setbacks, we must begin to think about their own reasons. Only objective and rational in the face of setbacks, success can slowly and slowly develop their ability to cope with setbacks, upgrade themselves. When their setbacks, the students can write down specific setbacks, and analyze, analyze the reasons why they fail, they can improve the behavior by which, combined with their own specific real situation, make a plan. Only by correctly understanding and analyzing setbacks, frustrations become to make their own power.

Experience and ability to deal with frustration community college students is not enough, it is prone to negative emotions. At this time a normal phenomenon, if the processing can help students get to develop positive emotions. When university students suffer setbacks negative emotions, you can cry, like eating food, exercise to divert attention, to regulate their emotions, so as to gradually develop a positive attitude to face difficulties.

Refers to a social interaction in the community commonly recognized norms and standards of behavior, this kind of interaction due to different individual social status and play different roles, social relations involving a variety of political, economic, and cultural aspects of life complex relationship, which includes kinship and relationships. Good social relations of college students is significant, it can give students adequate social support in college students suffered setbacks give the necessary assistance.

Students of social relations are the most important and most basic is the family relationship. In family relations, parental responsibility has more significant meaning. As children get older, parents should slowly change their attitude towards the child, from the child's point of view to more thinking, more consideration to the child's feelings, ask for more views of the child. As the child's parents and college students should be more exchanges, so that parents know that their thoughts and feelings. When parents disagree, try to calmly explain his views to the parents, I do not think that parents do not understand myself. When making important decisions, many ask the views of parents, trying to understand from their perspective, perhaps you find a better way. Only mutual respect, mutual understanding, family relationships Students will always be the most powerful support. 
Mainly refers to relationships other than family relations social relations. Student relationships are mainly friends, classmates and teachers composition. In establishing their relationships, college students should always do trust, forgiveness and gratitude. Trust in others and he is also trusted, which is conducive to the formation of a good intimate relationship, it is easy to enhance the well-being of College Students meal. Excuse me, is to allow others to make mistakes. Who make a mistake once, to forgive the fault of others give moderate, standing position of others to consider, it is the best way to maintain relationships. Thanksgiving, for those who helped themselves is grateful and try to help others. Thanksgiving allows college students in a positive attitude to see the world, enhance their sense of warmth and happiness.

\section{Conclusion}

Positive psychological qualities conducive to the growth of students' psychological health of college students develop good quality plays an important role in promoting. Students in culture positive psychological quality, to help students to establish positive cognitive ability, guide students to develop positive emotions to train students to establish a good social relation. Students thus effectively enhance the ability of anti-frustration, enhance the ability to deal with the problem of college students, to help students better adapt to an increasingly competitive society, better success, to obtain a sufficient sense of happiness.

\section{REFERENCE:}

[1] Dong Xue, Chen Li. Exploration Positive Psychology Perspective Psychological Quality Training System of College Students [J]. Shaanxi Education (Higher), 2015,01: 75 + 77.

[2] Luan Haiqing. Study of Positive Psychology Students Actively Psychology Cultivation Path [J]. Yangzhou University (Higher Education Study Edition), 2015,01: 62-65.

[3] Songguang Wen, Liu Qun. Students Actively Psychological Qualities: Domestic and Prospects [J]. Jinan Vocational College, 2015,05: 33-37.

[4] Zhao Liping, Pan Dening. Based on Positive Psychology Students Positive Psychological Quality Culture [J]. Journal Of Hebei Tourism Vocational College, 2013,03: 61-65.

[5] Zheng Peiliang. Research Training College Students' Positive Psychological Quality [J]. Inner Mongolia Agricultural University (Social Science Edition), 2013,05: 116-119.

[6] Gu Suhua. Thinking on Training Students' Positive Psychological Quality [J]. Inner Mongolia Normal University (Education Science Edition), 2014,07: 66-68.

[7] Lisi. Positive Psychological Quality Students Culture ～[J]. Education Forum, 2014,23: 178-179. 